\title{
Incidence and Risk Factors for Perianal Disease in Pediatric Crohn Disease Patients Followed in CEDATA-GPGE Registry
}

\author{
${ }^{*}$ Annecarin Brückner, ${ }^{*}$ Katharina J. Werkstetter, ${ }^{\dagger}$ Jan de Laffolie, ${ }^{\dagger}$ Claudia Wendt, ${ }^{*}$ Christine Prell, \\ ${ }^{\dagger}$ Tanja Weidenhausen, ${ }^{\dagger}$ Klaus P. Zimmer, and ${ }^{*}$ Sibylle Koletzko, the CEDATA-GPGE study group
}

\begin{abstract}
Objectives: Perianal disease (PD) with fistula and/or abscess formation is a severe complication in Crohn disease (CD). We examined prevalence, incidence, and risk factors for PD development in a pediatric CD cohort. Methods: Patients with CD from the prospective, multicenter registry for inflammatory bowel disease from Germany and Austria (CEDATA-GPGE) were included if diagnosed at the age of 18 years or younger, registered within 3 months after diagnosis, and having at least 2 follow-up visits within the first year of registration. We examined potential risk factors for PD with Kaplan-Meier analysis and a final Cox model considering sex, family history of inflammatory bowel disease, extraintestinal manifestations, disease location, and induction therapy (corticosteroids or nutritional therapy). Results: Of 2406 patients with CD, 742 fulfilled inclusion criteria (59\% boys, mean age at diagnosis $12.4 \pm 3.4$ years). PD was present at diagnosis in 41 patients $(5.5 \%$; $80.9 \%$ boys $)$, whereas 32 patients $(4.3 \%, 81.3 \%$ male $)$ developed PD during follow-up (mean $2.0 \pm 1.6$ years). The cumulative incidence of PD at 12 and 36 months after diagnosis was $3.5 \%$ and $7.5 \%$, respectively. Potential risk factors for PD development during follow-up were male sex (hazard ratio $=3.2$, [95\%; confidence interval 1.2-7.8]) and induction therapy with corticosteroids (hazard ratio $=2.5[1.1-5.5]$ ). Diagnostic evaluation at PD diagnosis was incomplete in $40 \%$ of affected subjects. PD resolved within 1 year in $50 \%$ of cases.

Conclusions: Approximately $10 \%$ of CD patients in our cohort suffered from PD within the first 3 years of their disease. Male sex and initial corticosteroid therapy were associated with an increased risk to develop PD after diagnosis.
\end{abstract}

Key Words: abscess, corticosteroids, incidence, perianal disease, prevalence

(JPGN 2018;66: 73-78)

Received October 16, 2016; accepted May 4, 2017.

From the *Division of Pediatric Gastroenterology, Dr. von Hauner Children's Hospital, Ludwig Maximilians University, Munich, and the †Department of Pediatrics, University Hospital Gießen and Marburg, Justus-Liebig-University, Gießen, Germany.

Address correspondence and reprint requests to Sibylle Koletzko, MD, $\mathrm{PhD}$, Dr. von Hauner Children's Hospital, Ludwig Maximilians University Medical Center, Lindwurmstr. 4, 80337 Munich, Germany (e-mail: sibylle.koletzko@med.uni-muenchen.de).

Supplemental digital content is available for this article. Direct URL citations appear in the printed text, and links to the digital files are provided in the HTML text of this article on the journal's Web site (www.jpgn.org).

The CEDATA-GPGE registry was funded by the Ein Herz für Kinder (A Heart for Children) Foundation and the Falk Foundation. This study was in part supported by a grant from MSD Sharp \& Dohme GmbH received by S.K., which had no influence on design and analysis of the study and interpretation of the data.

The authors report no conflicts of interest.

Copyright (C) 2017 by European Society for Pediatric Gastroenterology, Hepatology, and Nutrition and North American Society for Pediatric Gastroenterology, Hepatology, and Nutrition

DOI: 10.1097/MPG.0000000000001649

\section{What Is Known}

- Perianal disease, defined as fistula and/or abscess formation, is a severe complication and difficult to treat.

- The reported prevalence of perianal disease in newly diagnosed pediatric patients with Crohn disease varies from $8 \%$ to $15 \%$. Few data are available on incidence after diagnosis.

- Corticosteroids do not induce mucosal healing in Crohn disease.

\section{What Is New}

- Prospective data from a large registry on pediatric Crohn disease patients $(n=2406)$ indicate a prevalence of perianal disease at diagnosis of $5.5 \%$ with further $7.5 \%$ of subjects developing this complication within 3 years.

- Boys have a 3 times higher risk for developing perianal disease than girls.

- Use of corticosteroids as induction therapy after diagnosis is significantly associated with the development of perianal disease during follow-up. 
follow-up, and to determine potential risk factors for the development of PD in an unselected group of pediatric patients with CD followed in the registry of the Society for Pediatric Gastroenterology and Nutrition (CEDATA-GPGE). A secondary aim was to investigate whether physicians in Germany and Austria follow the guidelines for evaluation and management of $\mathrm{PD}$ in their patients.

\section{MATERIALS AND METHODS}

We retrospectively analyzed data of patients with $\mathrm{CD}$, which were registered from July 2004 to April 2014 in the German/ Austrian pediatric inflammatory bowel disease (IBD) patient registry CEDATA-GPGE. The CEDATA-GPGE registry is a prospective, multicenter registry in Germany and Austria, established since 2004 by the GPGE. The reporting institutions range from large specialized outpatient clinics at academic centers to small regional hospitals (14).

Reporting physicians were asked to complete a baseline case record form (CRF) and the first follow-up CRF reporting the results of diagnostic work up by the time a patient was included into the registry. The baseline CRF includes general patient characteristics, different sign and symptoms before and/or at the time of diagnosis, date and type (CD, ulcerative colitis, IBD-U) of final diagnosis, extraintestinal manifestations (EMs), and family history for IBD. This questionnaire has already obtained information about the presence of perianal fistula or abscess (see Supplemental Digital Content 1, http://links.lww.com/MPG/B8). The follow-up CRF includes data since the last documented visit on medical history including symptoms; stool behavior and EMs; anthropometrics, physical examination including perianal findings, laboratory parameters, diagnostic findings (endoscopy, histology, imaging), surgical procedures, disease localization, and severity; complications (fistula, abscess, stenosis, and other); comorbidities, and treatment (antibiotics, IBD-specific drugs, vitamins, and supplements). Exclusive enteral nutrition (EEN) as treatment option was only assessed from 2008 onwards in the registry.

Between 2004 and 2013, the institutions submitted patient's data via hard copy CRFs and since August 2013 online CRFs are in use. Because of financial constraints, enrollment of new patients was interrupted between 2010 and August 2013, whereas previously entered patients continued to have follow-up CRFs.

\section{Eligibility}

For this analysis we included patients with $\mathrm{CD}$ fulfilling the following criteria: age at initial $\mathrm{CD}$ diagnosis $\leq 18$ years, registry enrollment within 90 days after CD diagnosis with a completed baseline and first follow-up CRF, submission of at least 2 follow-up CRFs in the first year with a maximum time gap of 200 days between the consecutive follow-up CRFs. Eligible patients were censored, if the time interval between the consecutive follow-up CRFs after the first year was $>200$ days.

\section{Definitions}

PD was defined as perianal fistula and/or perianal abscess visualized during clinical examination and/or imaging. According to the perianal findings, we characterized 3 groups of patients:

1. Patients with PD at initial diagnosis had a documented PD at baseline and/or in the first follow-up CRF, documenting disease location and behavior at diagnosis (prevalence at diagnosis; group 1).

2. Patients developing PD during the course of disease had no PD documented at baseline and in the first follow-up CRF, but a documented PD thereafter (incidence of new PD after diagnosis; group 2).

3. Patients without PD had no documentation of PD in any CRF (no PD; group 3).

Family history of IBD included every occurrence of IBD in biological relatives of the patient. EMs included skin manifestations (erythema nodosum, pyoderma gangrenosum), fever $\left(>38.5^{\circ} \mathrm{C}>3\right.$ days without other focus), hepatobiliary (primary sclerosing cholangitis, autoimmune hepatitis, or overlap syndrome), pancreatic, renal or ophthalmological complications, arthralgia, or arthritis of peripheral joints and the spine. Disease location, rectal involvement, and disease behavior were extracted from the information given in the baseline and follow-up CRFs using the Paris classification (15). EEN therapy was defined as exclusive feeding with a balanced elemental or whole-protein formula for at least 4 weeks. Systemic corticosteroids as initial therapy included prednisone or methylprednisolone given orally or intravenously. Immune modulators included azathioprine (Aza), 6-mercaptopurine (6MP), and methotrexate.

Complete diagnostic evaluation for PD was considered with the following investigations performed: pelvic magnetic resonance imaging or trans-anorectal ultrasonography, colonoscopy or proctosigmoidoscopy, and manual examination under anesthesia by a colorectal surgeon $(11,12)$.

Diagnostic latency was defined as time between age at onset of symptoms and age of diagnosis. Physician global assessment regarding disease activity (remission, mild, moderate, or severe) was reported in the CRF at diagnosis and during follow-up (see Supplemental Digital Content 1, http://links.lww.com/MPG/B8 and Supplemental Digital Content 2, http://links.lww.com/MPG/B9). The short-Pediatric Crohn's Disease Activity Index (sPCDAI) (16) was calculated from the information provided on the CRFs. For discrimination between mild and moderate-severe disease activity a cut-off point of $\geq 30$ was set.

\section{Additional Questionnaire for Perianal Disease Management and Treatment}

For all identified patients with PD fulfilling the inclusion criteria, a questionnaire was sent to the respective institutions to confirm PD and provide additional information, particular regarding initial induction therapy, diagnostic and therapeutic management, and outcome of PD during follow-up.

\section{Statistical Considerations}

Descriptive analyses of the cohort and the 3 patient subgroups were given as percentages with mean \pm standard deviation (SD) or median (min; max) where appropriate. Chi-square test and the nonparametric Kruskal-Wallis test were applied for group comparison. Time until event was calculated by time in years from diagnosis of CD to first follow-up with documented PD, and in case of nonevent censored by last follow-up. Kaplan-Meier curves were performed and represent the survival distribution function of the event (PD). The cumulative incidence of PD is the difference of the curve to 1 and involved only patients with PD developing during follow-up (group 2) and patients without PD (group 3). We used Kaplan-Meier curves to compare incidental PD cases and patients without PD to identify potential risk factors for the development of PD regarding sex, positive family history for IBD, physician global assessment, C-reactive protein (CRP), sPCDAI (mild vs moderate/ severe), EM, age, disease location, rectal disease involvement at 
diagnosis, and corticosteroids or EEN within the first 3 months after diagnosis such as induction therapy. Variables were included if total missing was $<10 \%$. For the comparison of Kaplan-Meier curves, a nonparametric Log Rank test was selected.

For multiple analyses, Cox proportional hazards models were applied and estimated hazard rate ratios. To identify the potential risk factors, a backward selection was used starting with the potential risk factors recognized previously by Kaplan-Meier estimates (Log Rank with $P$ value $<0.05$ ) and fulfilled the proportional hazard assumption. Before starting selection procedure, all possible pairwise interactions were tested. The final model was conducted through stepwise backward elimination, based upon the Wald Chisquare test at the 0.05 level, or determined by clinical importance. The statistical significance was defined as $P<0.05$. The statistical software SAS Enterprise for Windows, Release 9.2 (SAS Institute, Cary, NC) was used for this study.

\section{Ethical Statement}

The ethical committee of the respective participating institutions approved the registry and all amendments. Age-appropriate informed consent was signed by children (starting at age 6) and their parents before inclusion. All data were submitted pseudonymized with respect to data security regulations.

\section{RESULTS}

In total, 4240 patients with IBD reported by 96 clinical institutions were included in the CEDATA-GPGE registry until March 2014 (55.1\% boys, median age at diagnosis of 13.7 years [range 0.3-22.9]); 2406 patients were diagnosed with CD $(58.4 \%$ boys; median age at diagnosis 13.9 years; range $0.2-22.9)$. Of the patients with $\mathrm{CD}, 742$ fulfilled all inclusion criteria and were reported by 70 of 96 participating clinical institutions with a median follow-up time of 1.2 years (range $0.2-7.3$ years). The most frequent reasons for exclusion were no registration within the first 3 months of diagnosis, no baseline and/or follow-up CRF reporting initial diagnostic workup, and/or no consecutive follow-up forms in the first year of registry enrollment (Fig. 1).

Out of 742 patients, 73 patients $(9.8 \%)$ were affected by $P D$, thereof with documented $\mathrm{PD}$ at initial CD diagnosis in 41 patients (5.5\%, group 1$)$, whereas 32 patients $(4.3 \%$, group 2$)$ developed $P D$ during follow-up with a median follow-up time in this registry of 1.6 years (range $0.2-7.0$ years). Of the 73 affected patients, 36 had

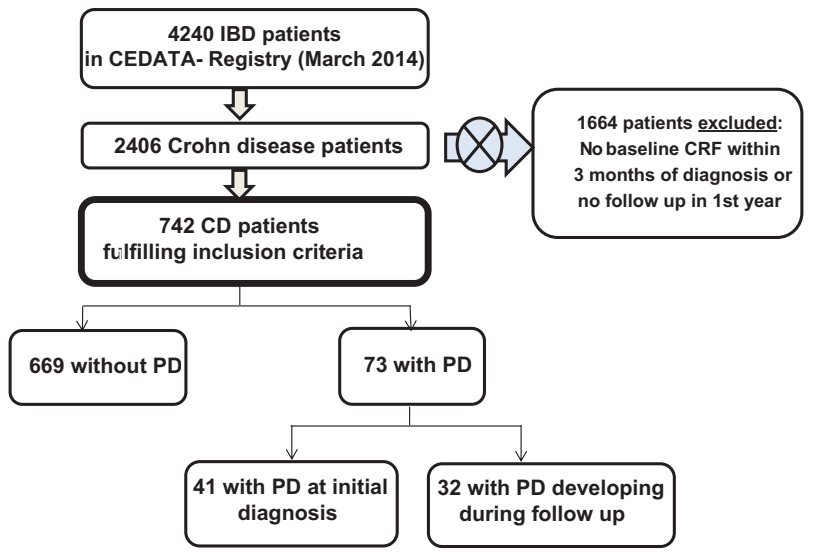

FIGURE 1. Flow chart of patients Inclusion - Final Cohort $(N=742)$. $\mathrm{CD}=$ Crohn disease; $\mathrm{CRF}=$ case record form; $\mathrm{IBD}=$ inflammatory bowel disease; $\mathrm{PD}=$ perianal disease. perianal fistula, 22 perianal abscess, and 15 patients were affected by both.

The cumulative incidence of new PD development after diagnosis was $3.5 \%$ at 1 year and $7.5 \%$ at 3 years, with 372 , 185 , and 77 patients followed at 1,2, and 3 years, respectively. Of 32 patients (group 2), 29 (90\%) developed PD within the first 1.5 years of initial diagnosis with a median time of 0.7 years (range, $0.2-4.6$ ). The cumulative incidence of $\mathrm{PD}$ was $9 \% 1$ year after CD diagnosis (groups 1 and 2).

Table 1 shows basic characteristics of the final cohort stratified by PD status in the 3 subgroups. Boys were more likely to be affected by PD than girls $(P=0.006)$. Rectal involvement at initial diagnosis was more frequent in patients of group $1(70.7 \%$; $29 / 41)$ compared to those in group $3(49.4 \% ; 330 / 669, P=0.01)$. There were no differences among patient groups regarding disease location, age at diagnosis, and positive family history for IBD.

Table 2 gives induction therapy used in the 3 subgroups and the total cohort. More than half of patients in this inception cohort $(55.6 \% ; 412 / 742)$ were initially treated with corticosteroids with a higher proportion in patients of group 2 developing PD during follow-up $(75.0 \% ; 24 / 32 ; P=0.02)$. EEN therapy was applied in 80 of $335(23.9 \%)$ patients enrolled after 2008 and in 4 of 10 of patients developing PD during follow-up. Aza at diagnosis was induced more often in patients developing PD than in those without PD (62.5\%; $\mathrm{N}=32$ vs $37.8 \% ; \mathrm{N}=669$, respectively, $P=0.0007)$.

Log rank tests in Kaplan-Meier indicated 2 following variables as potential risk factors for developing PD: male sex (logrank $=7.6 ; P=0.006)$ and the initial induction therapy with corticosteroids (log-rank $=5.5 ; P=0.018)$ (Fig. $2 \mathrm{~A}$ and $2 \mathrm{~B}$ ).

No significant associations with PD development after diagnosis were found for positive family history of IBD, EM, rectal disease involvement, age, physician global assessment, sPCDAI, and CRP at initial CD diagnosis. In a backward selection of the multiple Cox proportional hazards analysis, the effects of male sex and induction therapy with corticosteroids were confirmed as significantly related to $\mathrm{PD}$ development. By testing pairwise interactions between the given variables in the Cox regression, no effect modification was found.

Hazard rate ratios of the resulting risk factors revealed that the risk of developing PD was 3 times higher in boys than in girls (hazard ratio $=3.2,[95 \%$; confidence interval 1.2-7.8]). Induction therapy with corticosteroids within the first 3 months of diagnosis was associated with doubling the risk for occurrence of PD in pediatric patients with $C D$ (hazard ratio $=2.5[1.1-5.5]$ ).

\section{Additional Survey-Management of Perianal Disease}

The additional questionnaire on PD management was returned for 46 of 73 patients with PD; thereof 32 were diagnosed with $\mathrm{PD}$ at initial $\mathrm{CD}$ diagnosis. A complete diagnostic evaluation was performed in $57 \%(26 / 46)$ : magnetic resonance imaging in $67 \%$ (31/46), sigmoidoscopy or colonoscopy in $82 \%(38 / 46)$, and anal sonography in $11 \%(5 / 46)$ of patients. Information from the additional questionnaire confirmed the previous registry data, that two thirds of patients with PD developing during follow-up were treated with corticosteroids as induction therapy $(74 \% ; 34 / 46)$. After diagnosis of PD, a wide variety of drugs was initiated: half of patients received antibiotics (22/46) and/or Aza/6MP (26/46), $26 \%(12 / 46)$ were treated with biologicals, mostly infliximab (IFX), and $19 \%(9 / 46)$ were given systemic steroids. Surgical interventions were performed in 24 of 46 patients $(52 \%), 17$ of them had abscess incision, 9 fistula incision, 4 drainage with seton insertion, and 6 patients had at least 2 of those interventions combined. One patient 


\begin{tabular}{|c|c|c|c|c|}
\hline Patients characteristics at initial diagnosis & $\begin{array}{c}\text { Patients with PD at } \\
\text { diagnosis (group 1) } \\
(\mathrm{N}=41)\end{array}$ & $\begin{array}{l}\text { Patients with PD developing } \\
\text { during follow-up (group 2) } \\
\qquad(\mathrm{N}=32)\end{array}$ & $\begin{array}{l}\text { Patients with no } \\
\text { PD (group 3) } \\
(\mathrm{N}=669)\end{array}$ & $\begin{array}{l}\text { All patients } \\
(\mathrm{N}=742)\end{array}$ \\
\hline Age at diagnosis $($ mean $\pm \mathrm{SD})$ & $12.3 \pm 2.6$ & $13.4 \pm 2.8$ & $12.4 \pm 3.5$ & $12.3 \pm 3.3$ \\
\hline Male in \% & $78.1^{*}$ & $81.3^{*}$ & 56.6 & 59.0 \\
\hline Follow-up time in the registry in years (median, range) & $1.4(0.09-6.0)$ & $1.6(0.2-7.0)$ & $1.1(0.09-7.1)$ & $1.2(0.1-7.3)$ \\
\hline Positive family history for IBD in $\%$ & 12.2 & 15.6 & 17.0 & 16.6 \\
\hline $\begin{array}{l}\text { Extraintestinal manifestation within first } 3 \text { months } \\
\text { of diagnosis in \% }\end{array}$ & 24.4 & 40.6 & 28.7 & 28.9 \\
\hline \multicolumn{5}{|l|}{ Disease localization at baseline (Paris classification) in \% } \\
\hline L1 & 2.4 & 9.4 & 6.9 & 6.7 \\
\hline L1-L4 & 2.4 & 3.1 & 4.6 & 4.5 \\
\hline $\mathrm{L} 2$ & 4.9 & 12.5 & 13.3 & 12.8 \\
\hline L2-L4 & 19.5 & 9.4 & 8.2 & 8.9 \\
\hline L3 & 17.1 & 9.4 & 22.1 & 21.3 \\
\hline L3-L4 & 39.0 & 40.6 & 28.8 & 29.9 \\
\hline $\mathrm{L} 4$ & 2.5 & 0 & 1.2 & 1.2 \\
\hline Not available & 12.2 & 15.6 & 14.9 & 14.7 \\
\hline Rectal manifestation at diagnosis & $70.7^{*}$ & 56.3 & 49.4 & 50.9 \\
\hline Diagnostic latency in years (median, range) & $0.4(0.0-3.8)$ & $0.3(0.0-5.6)$ & $0.4(0.0-9.0)$ & $0.4(0.0-9.0)$ \\
\hline
\end{tabular}

$\mathrm{IBD}=$ Inflammatory bowel disease; $\mathrm{L} 1=$ involvement of the terminal ileum only, with limited or no cecal disease; $\mathrm{L} 2=$ shows only colonic involvement; L3 = small and large bowel involvement; L4 = upper GI involvement, isolated or combined with L1, L2, and L3; PD = perianal disease; SD = standard deviation.

${ }^{*} P<0.05$ versus group 3 .

received a colostomy. After 1 year of PD occurrence, complete healing was reported in $50 \%$ of the subcohort (22/44, 2 missing). These 22 patients received following treatment for PD: antibiotics $(59.2 \% ; 13 / 22)$, IFX in a combination with Aza/6MP (13.6\%; 3/22), Aza/6MP only $(13.6 \% ; 3 / 22)$, and no additional medication $(13.6 \%$; $3 / 22$ ). Surgical intervention was performed in 9 of 22 patients (41\%). Another 11 of 44 (25\%) patients recovered between 1 and 2 years, and further 6 after 2 years. In the remaining 5 patients $(12 \%)$, PD did not resolve until the end of this survey. Of those 5 patients, 3 had received biologics in combination with rectal steroids or Aza/6MP and 1 had additional surgical intervention with drainage. Another patient was treated with antibiotics, Aza/ $6 \mathrm{MP}$, nutrition therapy, and drainage. The last patient had no medical treatment but abscess incision. Information on relapses after initial healing was available in 37 of 46 patients. Relapses occurred in 7 of 37 patients in spite of treatment with antibiotics combined with Aza/6MP or methotrexate $(n=4)$, IFX combined with Aza/6MP and antibiotics $(n=1)$, or Aza/6MP only $(n=1)$. The last patient relapsed while not receiving any therapy.

\section{DISCUSSION}

In this pediatric cohort of 742 newly diagnosed $C D$ patients, approximately $10 \%$ experienced PD within the first 3 years after CD diagnosis. Boys had a 3 times higher risk for developing PD than girls. The use of corticosteroids as induction therapy after diagnosis was associated with the development of PD during follow-up.

Our cohort seems representative with similar age and sex distribution compared to all patients with $\mathrm{CD}$ in the registry. The male dominance is in concordance with figures reported from a large international prospective European registry (EuroKids) of 1221 newly diagnosed patients with IBD of which also $59 \%$ of the patients with CD were boys (17).

To avoid bias, we excluded patients with enrollment $>3$ months after initial CD diagnosis or $<2$ follow-up documentations

\begin{tabular}{|c|c|c|c|c|}
\hline $\begin{array}{l}\text { Induction therapy (within } 3 \text { mo } \\
\text { after diagnosis) in \% }\end{array}$ & $\begin{array}{c}\text { Patients with PD at } \\
\text { diagnosis }(\mathrm{N}=41) \text { (group 1) }\end{array}$ & $\begin{array}{l}\text { Patients with PD developing } \\
\text { during follow-up }(\mathrm{N}=32) \text { (group 2) }\end{array}$ & $\begin{array}{l}\text { Patients with no PD } \\
(\mathrm{N}=669)(\text { group } 3)\end{array}$ & $\begin{array}{l}\text { All patients } \\
(\mathrm{N}=742)\end{array}$ \\
\hline $\begin{array}{l}\text { Exclusive enteral nutrition from } \\
\text { January } 2008, N=10-335 \text { ) }\end{array}$ & 27.3 & 40.0 & 23.3 & 23.9 \\
\hline Corticosteroids & 65.9 & $75.0^{*}$ & 54.1 & 55.6 \\
\hline Antibiotics & $58.5^{\dagger, \ddagger}$ & $18.8^{\dagger}$ & 12.2 & 15.1 \\
\hline Methotrexate & 0.0 & 6.9 & 0.0 & 1.5 \\
\hline Immunomodulator (Aza/6MP) & $63.4^{*}$ & $62.5^{*}$ & 37.8 & $40.3 \%$ \\
\hline Infliximab & 2.5 & 3.5 & 1.8 & 1.9 \\
\hline
\end{tabular}

Aza $=$ azathioprine; $6 \mathrm{MP}=6$-mercaptopurine; $\mathrm{PD}=$ perianal disease.

* Significant $<0.05$ versus group 3 .

${ }^{\dagger}$ Significant $<0.0001$ versus group 3 .

${ }^{\ddagger}$ Significant $<0.05$ group 1 versus group 2 . 

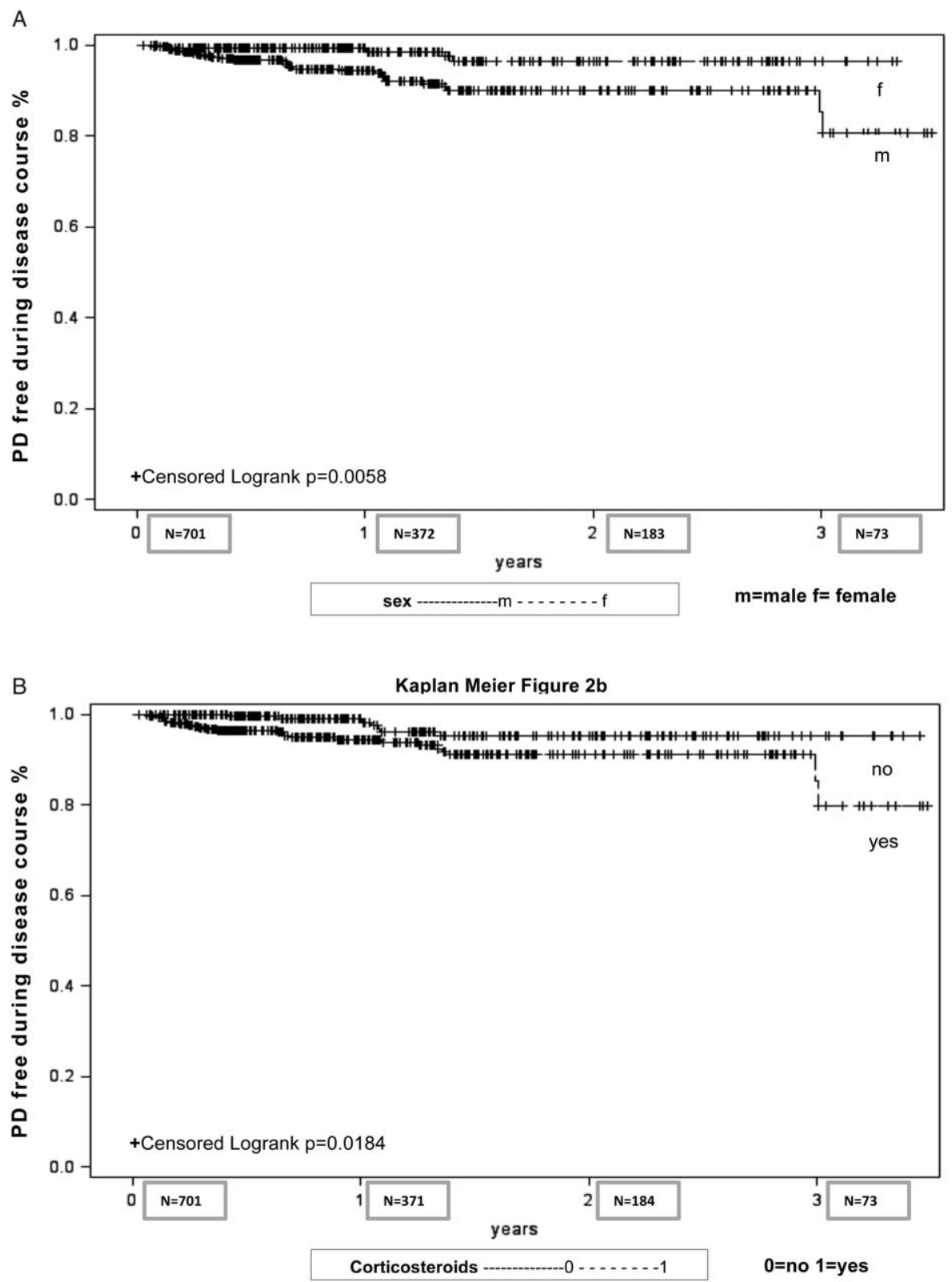

FIGURE 2. A, Kaplan-Meier-PD development stratified by sex in pediatric patients with Crohn disease $(C D)$ over time in years $(N=701)$. B, Kaplan-Meier-PD development stratified by induction therapy with corticosteroids within the first 3 months after diagnosis in pediatric patients with $C D$ over time in years $(N=701) .0=$ no induction therapy with corticosteroids; 1 =yes, induction therapy with corticosteroids; $\mathrm{F}=$ female; $\mathrm{m}=$ male; $\mathrm{PD}=$ perianal disease.

in the first year. The prevalence of PD at diagnosis (5.5\%) in our cohort is lower compared to data from the EuroKids registry $(9 \%)$ (17), from the Pediatric IBD Collaborative Group (10\%) (18), and the EPIMAP registry $(9 \%)(8)$ covering all patients with IBD from a defined region in Northern France (472 pediatric patients with CD diagnosed at an age $<16$ years between 1988 and 2002). Singer et al (7) performed a retrospective chart review of pediatric patients with $\mathrm{CD}$ seen at their institution during a 9-year period and reported a prevalence of perianal fistula in 40 of $318(13 \%)$ patients at time of diagnosis. A selection bias of more complicated cases reported from large IBD centers cannot be excluded. Our patients were diagnosed in 70 reporting sites including small hospitals and practices. A varying diagnostic latency due to different access to health care resources and specialists in different countries may also explain differences in the cohorts.

In our cohort, the cumulative incidence of PD at 1 year after diagnosis was $9.0 \%$ including prevalent cases at diagnosis. Gupta et al (9) reported a similar incidence of penetrating disease of $8.2 \%$ at 12 months after diagnosis $(\mathrm{N}=989)$, also including patients with the event at diagnosis. This cohort, however, focused on penetrating complications, not only on perianal abscess and fistula. The French registry EPIMAP observed a cumulative incidence of PD of $27 \%$ from diagnosis during a median follow-up of 7 years (8). The recent retrospective analysis from the "ImproveCareNow" registry reported PD prevalence at diagnosis of $9 \%$ with further $21 \%$ developing PD at any time point during follow-up resulting in a 
cumulative incidence of $30 \%$ after 6 years disease duration (10). Data from adult cohorts in the United States $(\mathrm{N}=179)$ and New Zealand $(\mathrm{N}=715)$ reported a $20 \%$ to $28 \%$ cumulative incidence of developing PD within 20 years of initial CD diagnosis, including patients with PD at diagnosis $(2,3)$. Since no such long-term data from pediatric-onset $\mathrm{CD}$ on the development of $\mathrm{PD}$ are available, it still remains unresolved, whether age of onset has an influence on developing this complication over time. Our data did not show any association between age at initial diagnosis and PD within the pediatric range.

Disease location did not differ between patients with PD at diagnosis, PD developing during follow-up, and no PD. A high percentage $(71 \%)$ of patients with $\mathrm{PD}$ at diagnosis had rectal involvement, which confirms data in adults (1). No significant difference was found between children who developed PD during follow-up and patients with no PD (both 47\%). We also found no association between family history for IBD, EMs, sPCDAI, CRP, and the presence of PD. EMs at initial diagnosis occurred in $29 \%$ of our cohort which is similar to other pediatric CD studies $(8,19,20)$.

Boys had a 3 times higher risk for developing PD than girls. Adler et al (10) reported an odds ratio of 1.49 for PD in boys compared to girls, which remained significant after adjustment $(P=0.013)$. Corticosteroids as initial induction therapy was associated with doubling the risk for developing PD. Pediatric guidelines for $\mathrm{CD}$ recommend either corticosteroids or EEN as induction therapy in moderate to severe CD. More than half of the cohort received systemic corticosteroids as induction therapy. It cannot be excluded that corticosteroids may have been used as initial therapy in children with more severe manifestation. The higher frequency of Aza treatment in patients with PD may point in the same direction.

Corticosteroids are less likely to induce mucosal healing compared to EEN and anti-TNF-alpha agents (21-23). Corticosteroids are potential risk factors for infections including abscesses. Although biological plausible, only randomized intervention trials will confirm a causal relationship between corticosteroids and the risk for developing PD.

The additional survey on PD management showed that IFX therapy was rarely used as initial therapy. This drug was licensed at first in September 2007. By that time, almost $50 \%$ of the cohort was already included in the registry. This may explain why only $26 \%$ of children with PD received biologicals, which are now recommended as first-line therapy in conjunction with surgical interventions (24). Antibiotics were the most commonly used medication for the treatment of PD, whereas surprisingly $20 \%$ received steroids.

Strength of the present study was the high number of unselected newly diagnosed patients with CD submitted by different types of clinical settings ranging from small standard care hospitals to university clinics and followed prospectively. The cumulative incidence may be biased due to a short patient follow-up time with a median of 1.2 years in the complete cohort and 1.6 years in patients who developed PD during disease progress.

Our data are relevant for the management of pediatric CD Because the majority of incident PD occurred within the first 18 months of follow-up close monitoring regarding PD development is warranted after diagnosis in pediatric patients with $\mathrm{CD}$, particularly boys. Further studies are needed to decide whether initial induction therapy with EEN is superior to corticosteroids to reduce the risk for PD development.

Acknowledgments: The authors thank Mrs. B. Filipiak (München) and Dr. A. Crispin (München) for statistical guidance and all reporting physician from the CEDATA-GPGE registry who reported their patients and the patients and their families.

\section{REFERENCES}

1. Hellers G, Bergstrand O, Ewerth S, et al. Occurrence and outcome after primary treatment of anal fistulae in Crohn's disease. Gut 1980;21:525-7.

2. Schwartz DA, Loftus EV Jr, Tremaine WJ, et al. The natural history of fistulising Crohn's disease in Olmsted County, Minnesota. Gastroenterology 2002;122:875-80.

3. Eglinton TW, Barclay ML, Gearry RB, et al. The spectrum of perianal Crohn's disease in a population-based cohort. Dis Colon Rectum 2012;55:773-7.

4. Kugathasan S, Judd RH, Hoffmann RG, et al. Epidemiologic and clinical characteristics of children with newly diagnosed inflammatory bowel disease in Wisconsin: a statewide population-based study. $J$ Pediatr 2003;143:525-31.

5. Palder SB, Shandling B, Bilik R, et al. Perianal complications of pediatric Crohn's disease. J Pediatr Surg 1991;26:513-5.

6. Markowitz J, Daum F, Aiges H, et al. Perianal disease in children and adolescents with Crohn's disease. Gastroenterology 1984;86:829-33.

7. Singer AA, Gdepalli SK, Eder SJ, et al. Fistulizing Crohn's disease presenting after surgery on a perianal lesion. Pediatrics 2016;137:e20152878.

8. Vernier-Massouille G, Balde M, Salleron J, et al. Natural history of pediatric Crohn's disease: a population-based cohort study. Gastroenterology 2008;135:1106-13.

9. Gupta N, Bostrom AG, Kirschner BS, et al. Incidence of stricturing and penetrating complications of Crohn's disease diagnosed in pediatric patients. Inflamm Bowel Dis 2010;16:638-44.

10. Adler J, Dong S, Eder SJ, et al. Perianal Crohn's disease in a large multicenter pediatric collaborative. J Pediatr Gastroenterol Nutr 2017;64:e117-24

11. Van Assche G, Dignass A, Reinisch W, et al., for the European Crohn's and Colitis Organisation (ECCO). The second European evidence-based consensus on the diagnosis and management of Crohn's disease: special situations. J Crohns Colitis 2010;4:63-101.

12. Amil-Dias J, Kolacek S, Turner D, et al. Surgical management of Crohn disease in children - guidelines from the Paediatric IBD Porto Group of ESPGHAN. J Pediatr Gastroenterol Nutr 2017;64:818-35.

13. Lewis RT, Maron DJ. Anorectal Crohn's disease. Surg Clin North Am 2010;90:83-97.

14. Buderus S, Scholz D, Behrens R, et al. Inflammatory bowel disease in pediatric patients: characteristics of newly diagnosed patients from the CEDATA-GPGE Registry. Dtsch Arztebl Int 2015;112:121-7.

15. Levine A, Griffiths A, Markowitz J, et al. Pediatric modification of the Montreal classification for inflammatory bowel disease: the Paris classification. Inflamm Bowel Dis 2011;17:1314-21.

16. Kappelman MD, Crandall WV, Colletti RB, et al. A short pediatric Crohn's disease activity index for quality improvement and observational research. Inflamm Bowel Dis 2011;17:112-7.

17. De Bie CI, Paerregaard A, Kolacek S, et al. Disease phenotype at diagnosis in pediatric Crohn's disease: 5-year analyses of the EUROKIDS registry. Inflamm Bowel Dis 2013;19:378-85.

18. Keljo DJ, Markowitz J, Langton C, et al. Course and treatment of perianal disease in children newly diagnosed with Crohn's disease. Inflamm Bowel Dis 2009;15:383-7.

19. Dotson JL, Hyams JS, Markowitz J, et al. Extraintestinal manifestations of pediatric inflammatory bowel disease and their relation to disease type and severity. J Pediatr Gastroenterol Nutr 2010;51:140-5.

20. Van Limbergen J, Russell RK, Drummond HE, et al. Definition of phenotypic characteristics of childhood-onset inflammatory bowel disease. Gastroenterology 2008;135:1114-22.

21. Mantzaris GJ, Christidou A, Sfakianakis M, et al. Azathioprine is superior to budesonide in achieving and maintaining mucosal healing and histologic remission in steroid-dependent Crohn's disease. Inflamm Bowel Dis 2009;15:375-82.

22. Canani BR, Terrin G, Borreli O, et al. Short- and long-term therapeutic efficacy of nutritional therapy and corticosteroids in paediatric Crohn's disease. Dig Liver Dis 2006;38:381-7.

23. Froslie KF, Jahnsen J, Moum BA, et al. Mucosal healing in inflammatory bowel disease: results from a Norwegian population-based cohort. Gastroenterology 2007;133:412-22.

24. Ruemmele FM, Veres G, Kolho KL, et al. Consensus guidelines of ECCO/ESPGHAN on the medical management of pediatric Crohn's disease. J Crohns Colitis 2014;8:1179-207. 